\title{
Anatomie Et Histologie De L'hépatopancréas Chez La Femelle Du Poisson, Chloroscombrus Chrysurusde Linnaeus, 1766 (Carangidae) En Côte d'Ivoire
}

\author{
Jean-Jacques Miessan, \\ Marie-Anne D'almeida, \\ Komenan Daouda Kouassi,
}

Laboratoire de Biologie Cellulaire,UFR Biosciences-Université Félix

Houphouët Boigny, Abidjan, Côte d'Ivoire.

\author{
Sylvain Yao, \\ Valentin N'douba,
}

Laboratoire d'Hydrobiologie, UFR Biosciences-Université Félix Houphouët

Boigny, Abidjan, Côte d'Ivoire.

\section{Goueh Gnahoue,}

Laboratoire de Biochimie et de Microbiologie,

École Normale Supérieure (ENS), Abidjan, Côte d'Ivoire.

Doi: 10.19044/esj.2018.v14n18p403 URL:http://dx.doi.org/10.19044/esj.2018.v14n18p403

\begin{abstract}
Chloroscombrus chrysurus is a pelagic fish species that belongs to the Carangidae family. It has a fairly high level of exploitation in Côte d'Ivoire. This paper focuses on the study of the maturity scale, the differentiation, and the histology of hepatopancreas. The methodological approach consisted of a macroscopic description of the hepatopancreas according to the six stages of sexual maturity. The microscopic study consisted of fixing the hepatopancreas, dehydrating them, impregnating them, including them, and making the sections that will be stained for optical microscopic observation. Macroscopic analysis has established six stages of sexual maturity in the species during which the growth and differentiation of hepatopancreas occur. From the juvenile to the adult stage, the size and color of the hepatopancreas vary. On the other hand, its aspect and shape remain unchanged. The hepatopancreas consists of two unequal lobes. The left hepatic lobe is always more developed than the right. On the microscopical level, among the female gender, the hepatopancreas is a mixed organ which constituted the hepatic parenchyme and pancreas. The pancreas differs in the hepatic parenchyma which is composed mainly of polygonal hepatocytes and blood vessels. The
\end{abstract}


hepatocytes are arranged in sinusoidal cords around the capillaries. The pancreas is composed of islets of Langerhans (endocrine pancreas) and serous acini Pancreatic (exocrine pancreas). Both structures progressively invade the hepatic parenchyma. The hepatopancreas is an essential organ in the physiology of fish.

Keywords: Chloroscombrus chrysurus, female, hepatopancreas, anatomy, histology

\section{Résumé}

Chloroscombrus chrysurus est une espèce pélagique de la famille des Carangidae. Il présente un niveau assez élevé d'exploitation en Côte d'Ivoire. La présente étude porte sur l'étude de l'échelle de maturité, de la différenciation et de l'histologie de l'hépatopancréas. La démarche méthodologique a consisté à une description macroscopique de l'hépatopancréas en fonction des six stades de maturité sexuelle. L'étude microscopique a consisté à fixer les hépatopancréas, à les déshydrater, à les imprégner, à les inclure et à réaliser les coupes qui seront colorées en vue d'une observation au microscope optique. L'analyse macroscopique a permis d'établir chez l'espèce six stades de maturité sexuelle au cours desquelles se déroulent la croissance et la différenciation de l'hépatopancréas. Du stade juvénile au stade adulte, la taille et la couleur de l'hépatopancréas varient.Par contre, son aspect et sa forme reste inchangés. Il est constitué de deux lobes inégaux. Le lobe hépatique gauche est toujours plus développé que le lobe droit. Au plan microscopique, chez la femelle, l'hépatopancréas est un organe mixte constitué dupancréas et du parenchyme hépatique. Le pancréas se différencie au sein du parenchyme hépatique qui est composé essentiellement d'hépatocytes de formes polygonales et de vaisseaux sanguins.Les hépatocytes sont disposés en cordons sinusoïdes autour des capillaires.Le pancréas estconstitué des îlots de Langerhans (pancréas endocrine) et des acini séreux pancréatiques (pancréas exocrine).Les deux structuresenvahissent progressivement le parenchyme hépatique.L'hépatopancréas est un organe essentiel dansla physiologie du poisson.

Mots-clés : Chloroscombrus chrysurus, femelle, hépatopancréas, anatomie, histologie

\section{Introduction}

Les Poissons constituent une composante essentielle dans l'alimentation de la population ivoirienne, en particulier Chloroscombrus chrysurus, qui présente une très haute valeur culinaire d'une part et une très haute valeur commerciale d'autre part. De plus, sur le marché ivoirien, elle est 
l'une des espèces dont le prix est abordable et à la portée des populations ivoiriennes à faible revenu.

En Côte d'Ivoire,plusieurs travaux se rapportent aux espèces pélagiques. On peut citer entre autresceux de Fantodji (1987) et Bodji et al. (2011) qui se rapportentàPomadasys jubelini et ceux deSylla (2010) qui se rapportent à Trachinotus teraia. Selon la littérature, la majorité des espèces pélagiques appartiennent au plateau continental ivoirien. Parmi celles-ci, on distingue également Chloroscombrus chrysurus objet de la présente étude.Cette espèce, représente une part non négligeable dans les différents débarquements des pêcheries industrielles et artisanales.Suite aux informations recueillies auprès des commerçantes, Chloroscombrus chrysurus serait fortement appréciée et consommée par la population ivoirienne. De plus, elle serait exploitée depuis plusieurs décennies.Sa forte demande sur les marchés induit sa pêche intensive. La surexploitation de l'espèce, pourrait conduireà moyen ou à long termeà son extinction.C'est donc pour relever cet état de fait que les présentes études sont entreprises.

Pour faire face à une éventuelle pénurie, la maîtrise de sa biologie entre autre de son cycle reproductif est primordiale pour envisager une pisciculture ultérieure. Bien que présentant des intérêts nutritionnels, économiques appréciables en Côte d'Ivoire, cette espèce n'a fait l'objet d'aucune d'étude. Son cycle sexuel et sa reproduction sont méconnus.

Chez les poissons, le cycle reproductif implique un ensemble de processus physiologiques et comportementaux en rapport avec divers facteurs de l'environnement biotique et abiotique (Paugy \& Levêque, 1999). L'effort de reproduction occasionne d'importantes dépenses énergétiques soutenues par l'apport direct de nutriments mais surtout par l'utilisation de réserves préalablement constituées et stockées dans l'hépatopancréas. La maîtrise du cycle de reproduction de Chloroscombrus chrysurus passe par la connaissance de son organe de stockage énergétique, l'hépatopancréas nécessaire à sa gamétogénèse. La différenciation de l'hépatopancréas à lieu durant la maturité sexuelle de Chloroscombrus chrysurus.

Elles s'insèrent dans un volet général se rapportant à l'étude du cycle reproductif et à la gamétogénèse de Chloroscombrus chrysurus.

Le présent article se rapporte à l'échelle de maturité sexuelle, à l'étude anatomique et histologique de l'hépatopancréas dont la différenciation se déroule au cours de la maturité sexuelle chez la femelle.

\section{Matériel et méthodes}

\section{Matériel biologique}

L'étude a porté sur 100 spécimens femelles de Chloroscombrus chrysurus. L'échantillonnage a été faite auprès des mareyeuses du port de pêche d'Abidjan d'une part et des mareyeuses du marché d'Abobo-doumé 
d'autre part. Ces dernières s'approvisionnent toutes dans les stocks de captures commerciales du port de pêche d'Abidjan.

Au laboratoire, les animaux sont triés et séparés dans un premier temps en fonction de la taille. Chez cette espèce gonochorique, la distinction des sexes n'est faite qu'après dissection. Par conséquent, le sexe et la maturité sexuelle des individus ont été déterminés après dissection. Les échantillons de l'hépatopancréas ont été prélevés pour l'étude anatomique et histologique.

\section{Méthodes}

\section{Méthodes macroscopiques}

La détermination des stades de maturité sexuelle est basée sur l'observation des gonades.L'échelle de maturité sexuelle utilisée est celle proposée par Sylla (2010) chez Trachinotus teraia et qui a été adaptée aux observations sur le terrain.

Les critères macroscopiques d'appréciations des gonades sont: la coloration, la consistance, la forme, la vascularisation et le volume occupé par la gonade dans la cavité abdominale. L’observation a permis de séparer les mâles des femelles.

$\mathrm{Au}$ plan macroscopique, les critères retenus sont: l'observation macroscopique, les différentes mesures et les poids des poissons.

Les différentes mesures concernant la taille sont :

- $\quad$ La longueur totale (LT) en mm, qui est mesurée de l'extrémité du museau à celle de l'extrémité extrême d'une des nageoires caudales.

- $\quad$ La longueur standard (LS) en mm, qui est la mesure de l'extrémité du museau jusqu'à l'insertion de la nageoire caudale.

En ce qui concerne le poids, les valeurs mesurées sont :

- $\quad$ La masse (MT) au gramme près du poisson non éviscéré.

- $\quad$ La masse (Mev) au gramme près du poisson vidé (après éviscération et extractiondes appareils génitaux et digestifs).

Au niveau des différents stades, les hépatopancréas ont été prélevés.

Dans ce cas, les critères d'appréciation de cet organe sont: la couleur, le volume, la forme et la consistance.

Les mesures prises sur l'hépatopancréas sont : la longueur, la hauteur et la masse.

- $\quad$ La masse (Mh) au gramme près des deux lobesde l'hépatopancréas.

Toutes les mesures ont été effectuées à l'aide d'une règle graduée. Le poids a été obtenu à l'aide d'une balance de type TEFAL.

Après la dissection, l'hépatopancréas situé dans la cavité générale est mis à nu suite au dégagement des caeca pyloriques et des mésentères. Cet organe observé in toto, est par la suite prélevé pour les études histologiques. Les prises de vue sont effectuées par un appareil photographique OLYMPUS SZ-20. 


\section{Méthodes microscopiques}

\section{Technique Histologique}

Pour l'étude histologique, les manuels de référence sont ceux de (Humason, 1967; Martoja \& Martoja-Pierson, 1967; Gabe, 1968; Nezelof et al., 1972; Locquin \& Langeron, 1978).

Les différents fragments des lobes de l'hépatopancréas dès leurprélèvement sont fixés par immersion dans du formol à $10 \%$, déshydratés dans des bains d'alcool à degré croissant $\left(70^{\circ}, 90^{\circ}, 100^{\circ}\right)$ et pré-imprégnés dans du toluène. L'imprégnation proprement dite ainsi que l'inclusion ont été faites dans de la paraffine de type PARAFINA PARA HISTOLOGICA dont le point de fusion est compris entre $58^{\circ} \mathrm{C}$ et $60^{\circ} \mathrm{C}$. Des coupes de $7 \mu \mathrm{m}$ réalisées au microtome de type MICROM dans les blocs sont traitées et colorées à l'hémalun-éosine. Pour obtenir des coupes convenables sans déchirures, les blocs histologiques entamés ont été constamment trempés de 12 à 24 Heures dans un bac contenant de l'eau courante. Ce trempage permet la réalisation aisée des coupes. Cette opération a été réalisée chaque fois que les coupes s'effritaient pendant l'étape de la microtomie. Les coupes ont également été collodionnées (Collodion 4\% MERK) afin d'éviter leur décollement. Les observations et les photos ont été faites respectivement avec le microscope MOTIC et le photomicroscope OLYYMPUS CKX41.

\section{Résultats \\ Échelle de maturité}

L'échelle de maturité a été définie après dissection et observation de l'anatomie interne chez la femelle (Figure 1). Elle a été établie grâce à l'observation minutieuse des gonades et a permis de suivrela différenciation de l'hépatopancréas.

Chez la femelle, l'échelle de maturité comporte six stades après observation des ovaires. La couleur des ovaires passe du jaune au jaune orangé ou au jaune rougeâtre. La vascularisation des ovaires est plus prononcée au stade V. C'est à ces différents stades que se différencie l'hépatopancréas qui a été prélevé pour les études histologiques. 


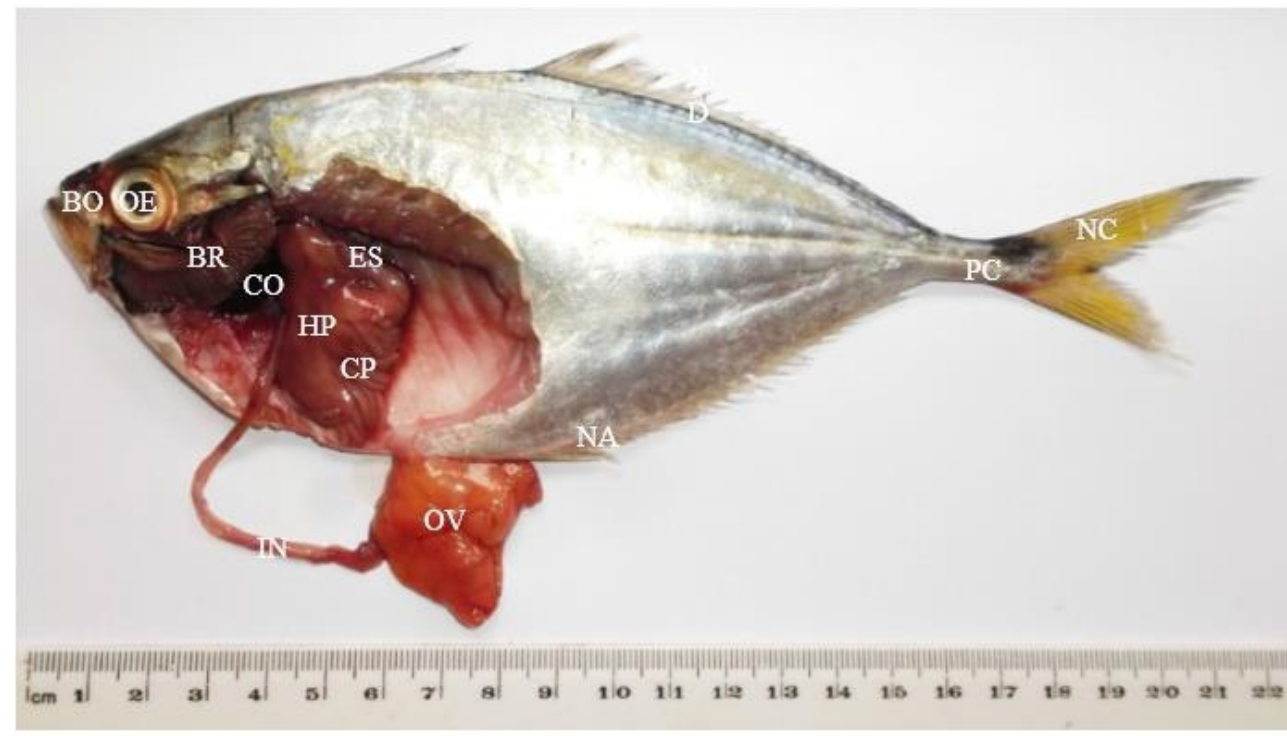

Figure 1: Morphologie et Anatomie de l'appareil reproducteur et digestif de la femelle de Chloroscombrus chrysurus.

BO: Bouche ; BR: Branchies; CO: Cœur; ES: Estomac; HP: Hépatopancréas; CP: Ceaca pyloriques; OV: Ovaires; NA: Nageoire anale; PC: Pédoncule caudal; ND: Nageoire dorsale; NC: Nageoire caudale; OE: Eil; IN: Intestin

\section{Différenciation de l'hépatopancréas chez laf emelle Chloroscombrus chrysurus \\ Différenciation macroscopique de l'hépatopancréas chez Chloroscombrus chrysurus}

Stade I : Le stade I correspond au stade immature. Ce stade regroupe tous les spécimens dont la longueur totale (LT) est comprise entre 13 et 15 $\mathrm{cm}$ (Figure 2A). L'hépatopancréas se présente sous une forme quelconque et de couleur marron brun (Figure 2A1). Il est ferme, aplatis et occupe le quart de la cavité abdominale (Figure 2A). Cet organe est constitué de deux lobes (Figure 2A1). Le lobe hépatique gauche est quatre fois plus volumineux que le lobe droit (Figure 2A1). La longueur est comprise entre 1 à $2,0 \mathrm{~cm}$ et l'épaisseur varie de 1 à $1,5 \mathrm{~cm}$. La masse des spécimens est comprise entre 31 et $50 \mathrm{~g}$, et celle de l'hépatopancréas oscille entre 0,25 et $0,50 \mathrm{~g}$.

Stade II : Il rassemble tous les spécimens qui ont une longueur totale comprise entre 15 et $16,5 \mathrm{~cm}$ (Figure 2B) et dont la masse varie de 51 à $65 \mathrm{~g}$. À ce stade, l'hépatopancréas occupe le tiers de la cavité abdominale et reste toujours ferme, aplatis et de teinte marron brun (Figures 2B et 2B1). La masse de l'hépatopancréas est comprise entre 0,50 à $0,85 \mathrm{~g}$.Sa longueur et son épaisseur varient peu par rapport au stade précédent (Figure 2B1).Le lobe hépatique gauche représente letriple du lobe droit (Figure 2B1). 
Stade III : Le stade III correspond au stade de puberté. Les individus, long de $18 \mathrm{~cm}$ (Figure 2C), ont une masse totale comprise entre 65 et $105 \mathrm{~g}$. Le volume de l'hépatopancréas varie peu par rapport au stade précédent (Figure 2C). Les caractéristiques de l'hépatopancréas sont les mêmes qu'aux stades précédents (Figure 2B), sauf que la couleur vire au rouge (Figure 2C1). L'hépatopancréas a une longueur comprise entre 2,5 et $3,0 \mathrm{~cm}$ avec une épaisseur qui se situe entre 1,5 et 2,5cm (Figure 2C1).La masse de cet organe varie de 0,85 à 1,50g. Le lobe hépatique droit est le tiersdulobe gauche (Figure 2C1).

Stade IV : Au stade IV, la longueur totale des spécimens et la hauteur de l'hépatopancréas varient très peu par rapport au stade III (Figure 2C).L'hépatopancréas se trouvant au terme de son développement reste ferme, volumineux et rougeâtre (Figure 2D1). Il a la même localisation dans de la cavité abdominale (Figure 2D). Le lobe gauche est deux fois plus volumineux que le lobe droit (Figure 2D1). Cette augmentation du lobe gauche est liée à la croissance du poisson. La masse des individus de ce stade est comprise entre 97 et 145g. Quant à celle de l'hépatopancréas, elle varie de 1,4 à 1,8g.

Stade V : Le stade V regroupe tous les spécimens dont la longueur totale est comprise entre 18,5 et $20 \mathrm{~cm}$ (Figure 2E). La masse des spécimens oscille entre 96 à $150 \mathrm{~g}$ et celle de l'hépatopancréas entre 1,25 et $2,25 \mathrm{~g}$. L'hépatopancréas occupe les $1 / 6$ de la cavité abdominale (Figure 2E). Lesdeux lobes de l'hépatopancréas sont approximativement identiques et de couleur marron (Figure 2E1). Il est aplati et ferme. L'hépatopancréas, long d'environ $3,5 \mathrm{~cm}$ à une épaisseur comprise entre 2 et $2,5 \mathrm{~cm}$ (Figure 2E1).

Stade VI : À ce stade, la taille des poissons varie entre 20 et $22 \mathrm{~cm}$. Leurs hépatopancréas mesurent en moyenne $4,0 \mathrm{~cm}$ et pèsent environ $1 \mathrm{~g}$. L'hépatopancréas se présente toujours sous une forme quelconque mais est par contre marron brun. Il devient flasque, aplatis et occupe le quart de la cavité abdominale. 


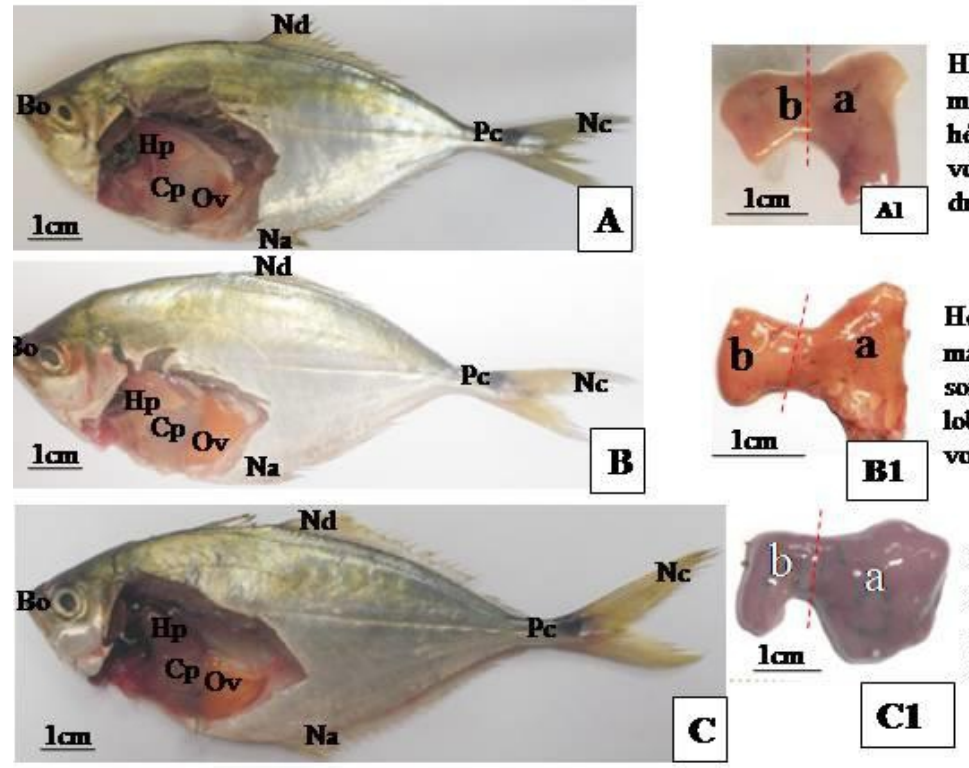

Hépatopancréns de conleur marron brun, Le lobe hépatique gauche est plus volumineax que le lobe droit.

Hépatopancréns de couleur marron brun, les deax lobes sont disproportionnels: le lobe ganche est plus voluminearx que le lobe droit.
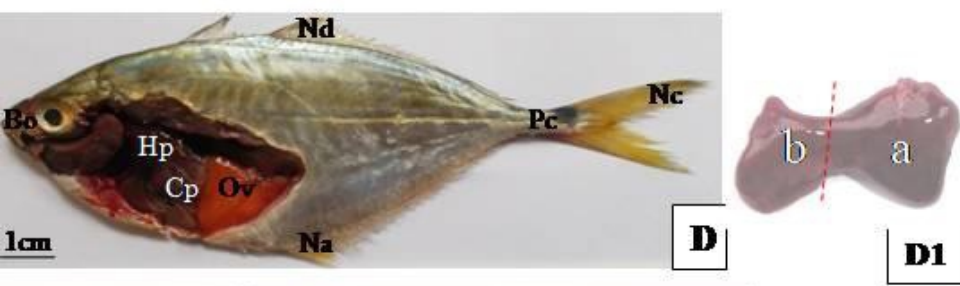

Hépatopancréns de conlear rongefitre, Le lobe gauche est développé

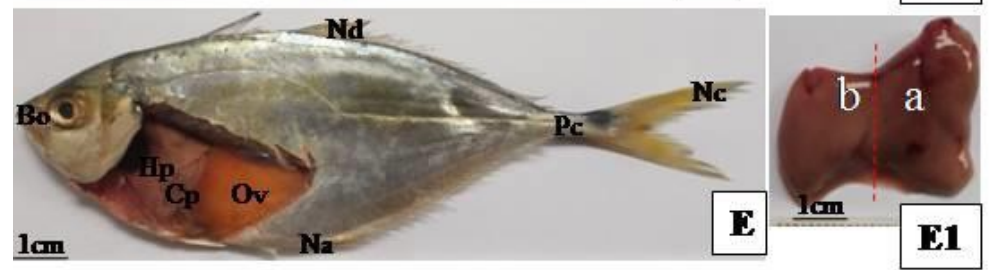

Hépatopancréns de Conleur rongen̂tre, les deax lobes Sont proportiomels.

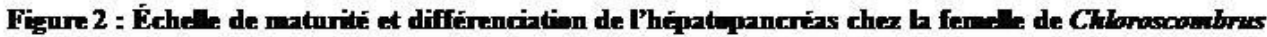
choswens

A: Femelle an stade I; A I: Hépatopancréss dela femelle an stade I- B : Femelle an stade II;

B2:Hépatopancréns dela femelle au stade II-C : Femelle au stade II-CI: Hépatopancréas dela femelle an stade III-D: Femelle an stade II; DI: Hépatopancrés dela femelle an stade IV; E : Femelle au stade II; EI : Hépatopancréns dela femelle an stade V.

Nc-Nageoire caudale, Na: Nageoire anale, Nd-Nageoire dorsale, Hp: Hépatopancréas, Cp; Ceaca pyloriques, Ov- Ovaire, Pc: pédoncule caudal, Bo: Bonche.

b-lobe hépatique droit, a: lobe hépatique gauche.

Étude histologique de l'hépatopancréas chez la femelle de Chloroscombrus chrysurus

Stade I et II : L'observation de l'hépatopancréas au stade immature (Figures 3A et 3B) révèle la présence du parenchyme hépatique et du pancréas. Le parenchyme hépatique organisé en lames hépatiques ne présente 
aucune caractéristique spécifique (Figures 3C à 3G). C'est un tissu d'aspect homogène dans lequel se trouvent des capillaires (Figure 3G). Le parenchyme hépatique est formé d'hépatocyte de formes polyédriques. Dans ce parenchyme, on distingue les artères hépatiquesde calibres variables (28 à 35 $\mu \mathrm{m}$ ) (Figures 3D et 3F). Quant au pancréas, il se différencie à l'intérieur du parenchyme hépatique. Il est constitué de deux parties : la portion exocrine (Figures 3C et 3E) et la portion endocrine (Figure 3G). Les deux parties sont éparpillées au sein du parenchyme hépatique. Le pancréas exocrine est constitué d'acini de type séreux et de canaux excréteurs (Figure 3E). Le pancréas endocrine est représenté par les îlots de Langerhans (Figure 3G) qui sont de petites formations sphériques. Ces îlots de Langerhans sont séparés du parenchyme hépatique par une capsule (Figure 3G).

Stade III : L'hépatopancréas des spécimens du stade III (Figure 4A), est caractérisé par des modifications structurales (Figures 4B à 4F). On note l'apparition de lames hépatocytaires constituées d'un ensemble d'hépatocytes regroupés entre eux. Ces lames, de taille variable ont une épaisseur comprise entre $20 \mu \mathrm{m}$ et $25 \mu \mathrm{m}$. On distingue également des capillaires sinusoïdes entre les lames hépatocytaires (Figures $4 \mathbf{C}$ à $4 \mathbf{E}$ ). Le calibre des différents vaisseaux sanguins a légèrement augmenté (Figures 3C et 4D). L'artère hépatique est constituée d'une lumière d'environ $40 \mu \mathrm{m}$ de diamètre et d'une paroi dont l'épaisseur moyenne est de $25 \mu \mathrm{m}$ (Figure 4C). Les veines portes d'environ $16 \mu \mathrm{m}$ de diamètre apparaissent et sont visiblesdans le parenchyme hépatique (Figure 4D). On distingue à ce stade, la formation des canaux pancréatiques (Figure 4F).

Stade IV : L'hépatopancréas (Figure 5A) est caractérisé par des changements morphologiques des deux éléments du pancréas à savoir les pancréas endocrine et exocrine (Figures $\mathbf{5 C}$ à $\mathbf{5 F}$ ). En effet, les îlots de Langerhans de forme arrondis au départ (environ $50 \mu \mathrm{m}$ de diamètre) s'allongent avec la différenciation de canaux excréteurs (Figures 5C à 5E). En ce qui concerne les canaux excréteurs du pancréas exocrine, ils sont limités par une paroi de $35 \mu \mathrm{m}$ d'épaisseur, entourant ainsi la lumière (Figure 5F). En revanche, le parenchyme hépatique présente les mêmes caractéristiques qu'aux stades précédents sauf que la lumière des capillaires sinusoïdes est importante et mesure en moyenne $10 \mu \mathrm{m}$ de largeur (Figures 5C à 5E).

Stade V : Au niveau de l'hépatopancréas (Figure 6A), on distingue des canaux pancréatiques et des îlots de Langerhans partiellement ou totalement vidés de leurs contenus cellulaires (Figures 6C à 5E). Ce tissu présente une désorganisation des lames hépatocytaires accompagnée d'une légère augmentation de la lumière des capillaires sinusoïdes (Figures $\mathbf{6 C}$ et 6D).

Stade VI : Au niveau du tissu, au stade VI, les lumières des capillaires semblent élargir.Leur diamètre varie de(40 à $50 \mu \mathrm{m})$ tandis que les lames 
hépatocytaires présentent un rétrécissement (Figures 7C à 7D). Elles semblent totalement vidées de leurs contenus perdant ainsi leurs configurations initiales (Figures 7B et 7D). Sur le parenchyme hépatique, on note la présence des centres mélanomacrophages (Figures 7B et 7C) représentés par des petitspoints noirsdisséminés sur tout le parenchyme.
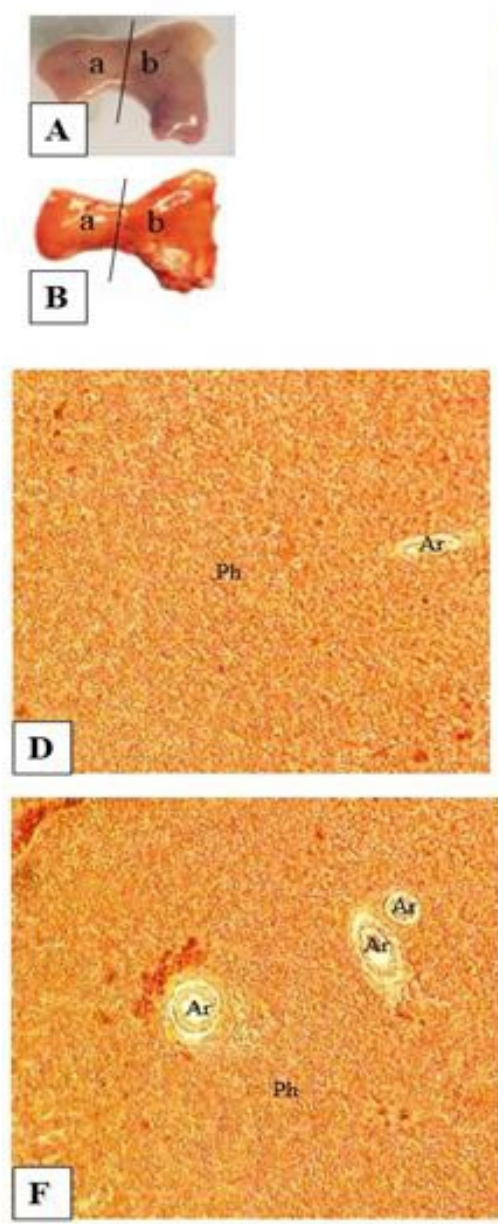

Figure 3: Anatomie et histologie de l'hépatopancréas chex la femelle de Chloroscombrus chrysurus aux stades I et IL.

A et B: Anatomie de 1'hépatopancréas; C: Vue d'ensemble d'une portion de la coupe de l'hépatopancréas; D: Détail d'une portion de l'hépatopaneréas montrant le parenchyme hépatique. E: Vue de la structure d'une portion du pancréas exocrine de la figure C. Le pancréas exocrine est constitué des acini de types séreux et des canaux excréteurs. Ces canaux sont entourés de tissu conjonctif. F: Détail de trois artères hépatiques de calibres différents disséminées dans le parenchyme hépatique. Les artères hépatiques sont constituées d'une lumière et d'une paroi. G: Vue détaillée de portion du parenchyme hépatique montrant le pancréas endocrine. Les îlots de Langerhans sont séparés du parenchyme hépatique par une capsule. Coloration: Hémalun-éosine. Grossissement: C: G X 40; D, E, F, G: G X 100 II: flot de Langerhans, C: Capillaires, Vp: Veine porte, Te: Tissu conjonctif, Ph: Parenchyme hépatique, Lh: Lame hépatocytaire, As: Acini séreux, Ce: Canal excréteur, $\mathrm{Cp}_{\mathrm{p}}$ : Capsule. 

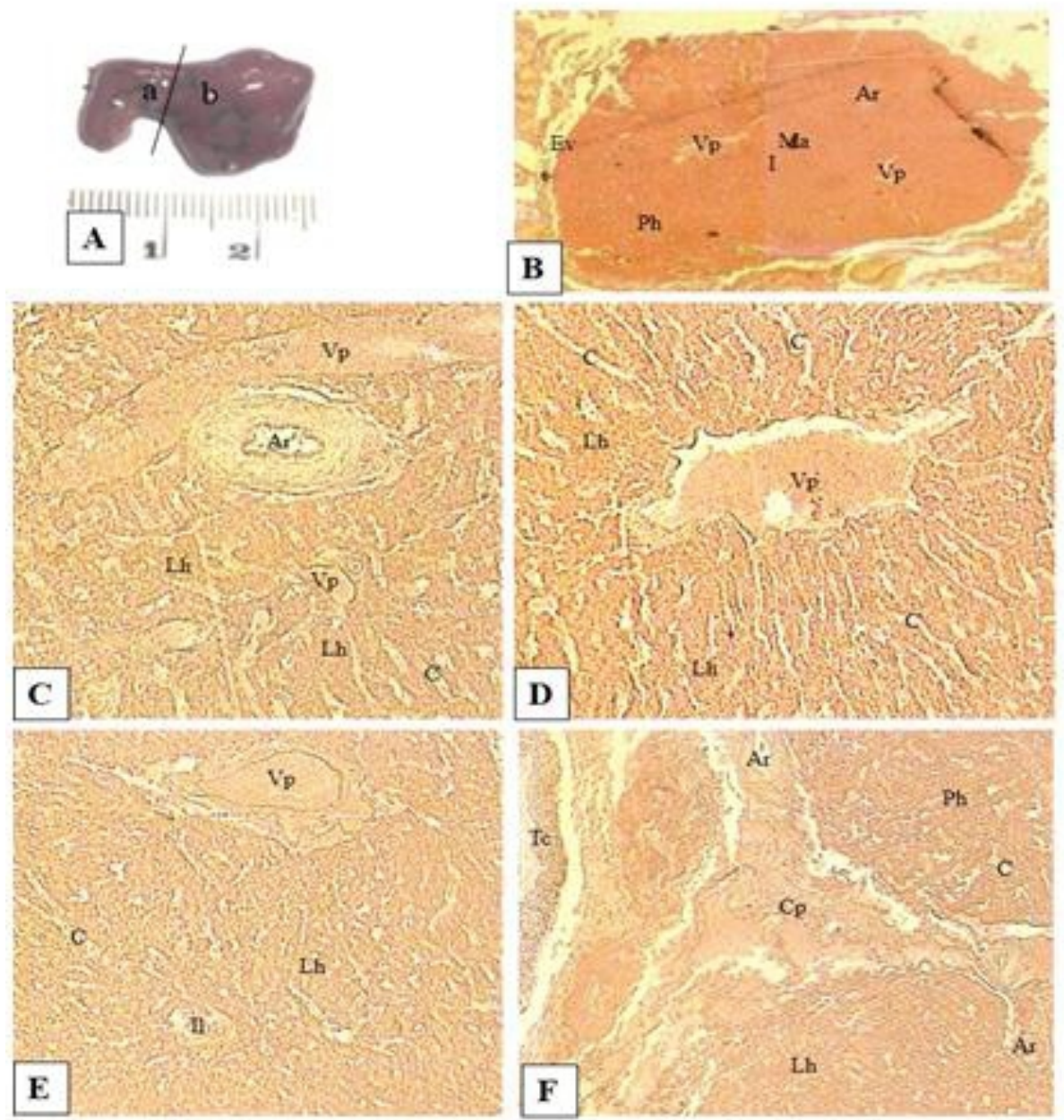

Figure 4: Anatomie et histologie de I'hépatopancréas de la femelle de Chloroscombrus chrysurus au stades III.

A: Anatomie de l'bépatopancréas; B: Vue d'ensemble d'uene portion de la coupe de l'hépatopancréas.

C: Détail des capillaires entre lames hépatocytaires. Un gros artère bépatique est visible près de ta veine porte. D: Détail d'une grosse veine porte disséminée dans le parrenchyme hépatique. E: Vue détaillée du parenchyme hépatique montrant la veine porte et les ilots de Langerhans.

F: Vue détaillée d'une portion d'bejpatopancréas présentant l'ebaoche et le développement des canaux pancréatiques.

Coloration: Hémalun-éosine, Grossissement: B: G X 40; C, D, E, F: G X 100.

In: fllot de Langerhans, V: Vieine porte, C: Capillaires, Te: Tissu conjonctif, Ph: Parenchyme hépatique, Lh: Lame bépatocytaire, Cp: Canaux pancrèatiques, Ev: Enveloppe, a: Lobe hẻpatique droit, b: Lobe hẻpatique gauche. 

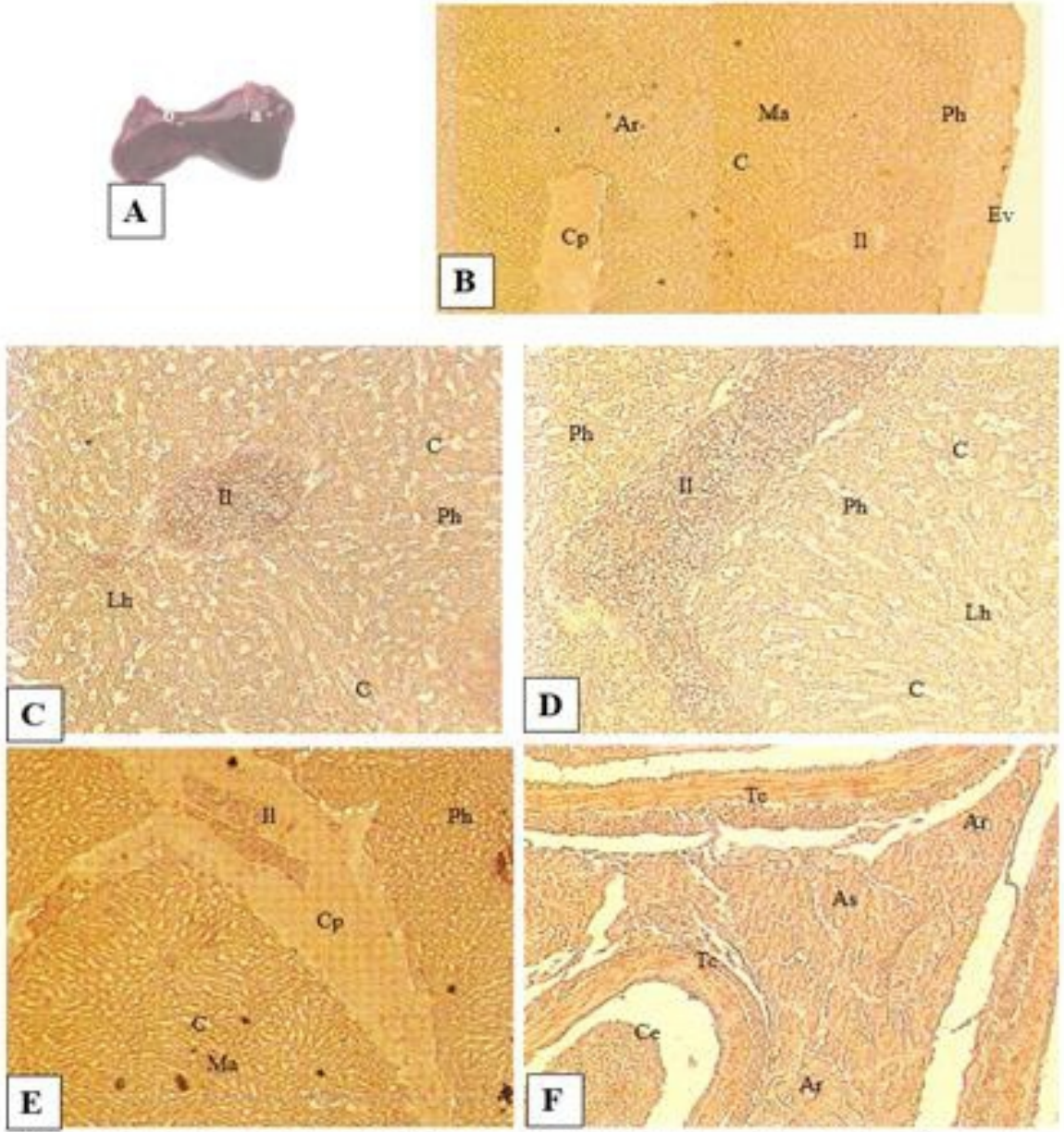

Figure 5: Anatomie et bistologie de l'hépatopancréas de la femelle de Caloroscombrus chersuras a stades $\mathrm{IV}$.

A: Anatomie de I' hépatopancréas; B: Vue d'ensemble d'une portion de la coupe d'bejatopancréas au stade

IV. C: Vue détaillée d'un Ilot de Langerhans de forme arroedis et volumineux au centre du parenchyme hépatique. D: Détail d'un long et gros llot de Langerhans dans le parenchyme hépatique. E: Détail d'un canal pancréatique bifurqué contenant des tlots de Langerhans. F: Vue détallée d'une portion du paneréas exocrine montrant les acini séreux entre les canaux excréteurs.

Coloration: Hèmalun éosine, Grossissement: B: G X 40; C, D, E, F: G X 100

II: Îlot de Langethans, C: Capillaises sinusoides, Ph: Parenchyme bépatique, Ev: Membrane, As: Acini séreux, Te: Tissa conjonctif, Ma: Cellule de Kupfer, Ce: Canal excréteur, $\mathbf{C p}$ : Canal pancréatique,

Ar: Artère hépatique, Lh: Lame hépatique, a: Lobe droit, b: Lobe gauche. 


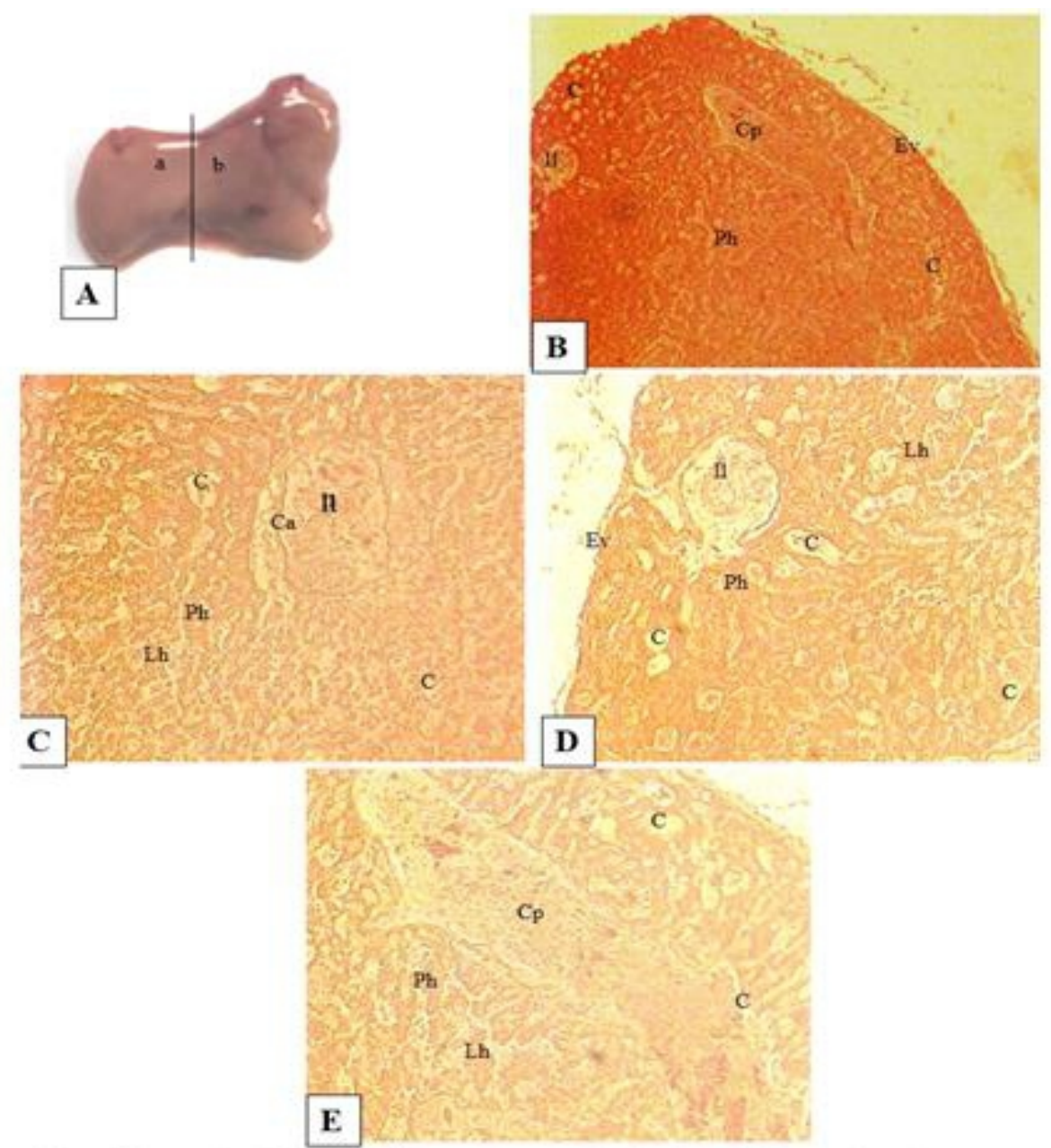

Figure 6: Anatomie et histologie de l'hépatopancréas de la femelle de Chloroscombrus cknsarus au stades $\mathrm{V}$.

A: Anatomie de l'hèpatopancréas; B: Vue d'ensemble d'une portion de la coupe de t'hépatopancréas;

C et D: Vue détaillée du parenchyme hépatique montrant des llots de Langerhans partiellement ou totalement vidés de leurs contenus. E: Détail d'une portion du canal pancréatique au sein du parenchyme bepatique.

Coloration: Hémalun éosine. Grossissement: B: G X 40; C, D, E: G X 100

II: ilot de Langerhans, C: Capillaires sinusordes, Ph: Parenchyme bèpatique, Cp: Canal pancréatique, Ev: Membrane, Lh: Lame hepatique, $\mathrm{Ca}_{\text {: }}$ Capsule, a: Lobe hépatique droit, b: Lobe hépatięue gauche. 


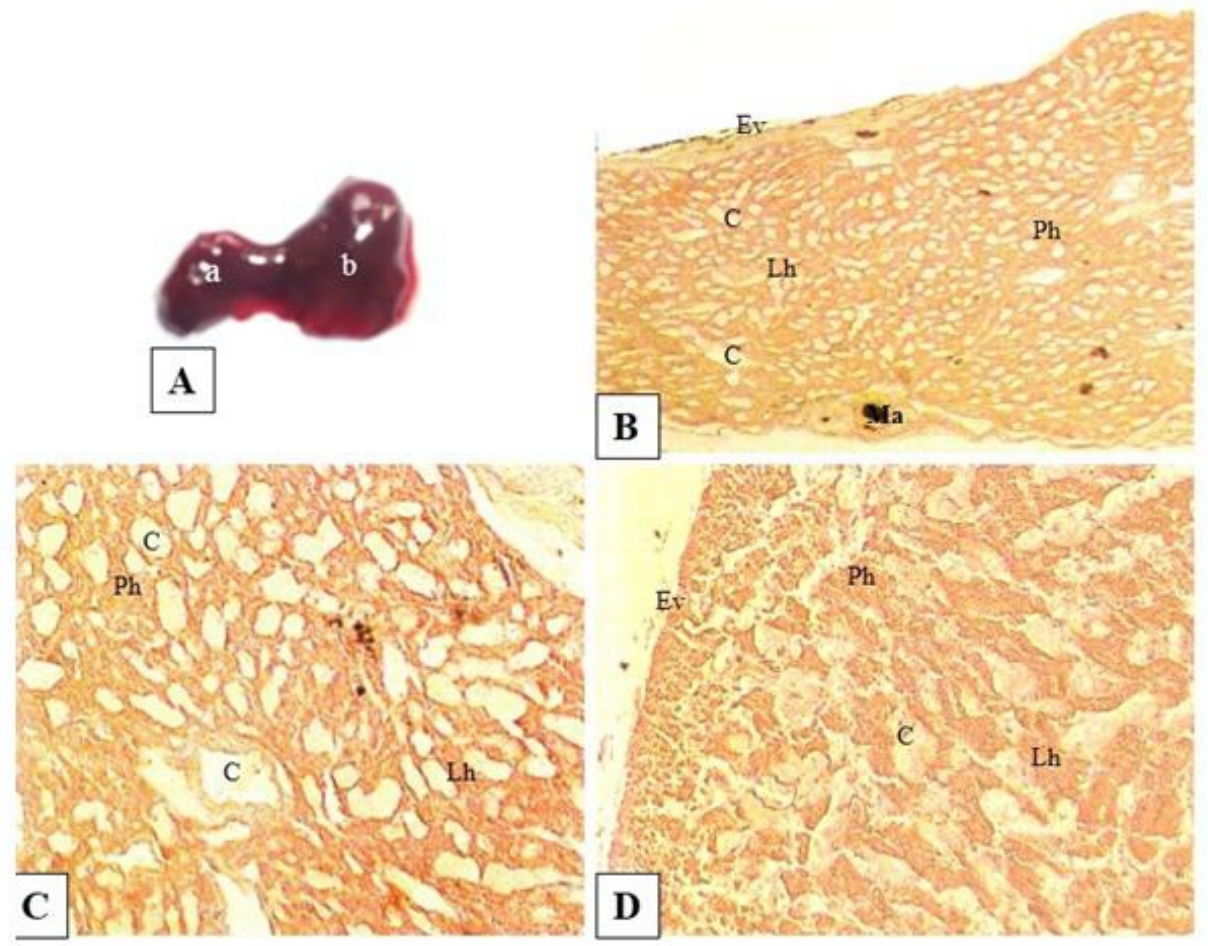

Figure 7: Anatomie et histologie de l'hépatopancréas de la femelle de Chloroscombrus chrysurus au stades VI.

A: Anatomie de l'hépatopancréas; B: Vue d'ensemble d'une portion de la coupe d'hépatopancréas; C et D: Détail du parenchyme hépatique montrant la désorganisation des lames hépatocytaires et l'élargissement de la lumière des capillaires.

Coloration: Hémalun-éosine. Grossissement: B: G X 40; C, D: G X 100.

Il: Îlot de Langerhans, C: Capillaires sinusoides, Ph: Parenchyme hépatique, Ev: Membrane, Ma: Cellule de Kupffer, Lh: Lame hépatocyte, a: Lobe hépatique droit, b: Lobe hépatique gauche.

\section{Discussion}

\section{Échelle de maturité sexuelle et différenciation de l'hépatopancréas}

D'après la présente étude, la maturité sexuelle de la femelle de Chloroscombrus chrysurus se fait suivant 6 stades. Les présents résultats sont en accord avec ceux des travaux antérieurs sur l'échelle de maturité sexuelle chez de nombreuses espèces des Poissons Téléostéens obtenus par (N'goran 1995 ; Sylla, 2010 ; Djadji et al., 2010 ; Bodji et al., 2011 ; Konan et al., 2013). Ce n'est pas le cas chez les espèces Pomadasys jubelini (Fantodji, 1987) et Distichodus rostratus (Berté et al., 2008), chez qui les travaux ont relevés respectivement 8 et 5 stades de maturité sexuelle. La variabilité des stades de maturité pourrait être due à l'organe de stockage énergétique et à l'activité de reproduction des espèces. L'échelle de maturité sexuelle, englobe 
à la fois les étapes de croissance des spécimens et de différenciation de l'hépatopancréas. La mensuration et la colorabilité de l'hépatopancréas ont été étudiées de façon concomitante avec l'échelle de maturité sexuelle de Chloroscombrus chrysurus. Le processus de différenciation de l'hépatopancréas tient compte de son aspect anatomique et de son organisation structurale au cours de la maturité sexuelle.Faute de références bibliographiques relatives à l'étude de l'hépatopancréas chez les Poissons, aucune comparaison n'a été possible dans l'embranchement des Poissons. En revanche, la discussion a été menée par rapport à l'anatomie, à l'histologie et à la physiologie déduite de la structure histologique de cet organe en référence aux Mammifères.

Les présentes études montrent que tous les individus dont la longueur de l'hépatopancréas est comprise entre 1,0 et $2,5 \mathrm{~cm}$ sont immatures. Ce groupe correspond aux individus du stade I et II. Il est de couleur marron brun. Les femelles pubères correspondant au stade III ont un hépatopancréas dont la longueur est comprise entre 2,5 et 3,0 cm et une épaisseur moyenne de 1,7 cm. À ce stade, l'hépatopancréas est rouge. Les spécimens matures sont ceux des stades IV, V et VI. L'hépatopancréas de ces poissons, ont une longueur comprise entre $3,5 \mathrm{~cm}$ et $4,0 \mathrm{~cm}$ et une épaisseur moyenne de $1,4 \mathrm{~cm}$. Aucune référence relative à ces caractéristiques dans la littérature ne vient étayer les présents résultats.

\section{Aspect anatomique de l'hépatopancréas}

$\mathrm{Au}$ plan anatomique, l'hépatopancréas de la femelle de Chloroscombus chrysurus est une masse qui est constitué de deux lobes inégaux, un grand lobe hépatique gauche et un petit lobe droit. Ce résultat concorde avec ceux de Choulika (2002) qui ont montré que le foie des Téléostéens est constitué de deux lobes. La couleur de l'hépatopancréas passe du marron brun au stade juvénile à la couleur rouge vif au stade adulte. La variation de sa coloration pourrait s'expliquer par son activité physiologique et par son régime alimentaire. En effet, selon Genten et al. (2011), la variation de la couleur du foie s'explique par un changement de régime alimentaire. En outre, Choulika (2002) et Genten et al. (2011)ont indiqué que chez les poissons sauvages, le foie est généralement brun rougeâtre chez les carnivores ou brun clair chez les herbivores. Durant la différenciation de l'hépatopancréas, la taille, la forme et le volumeaugmentent durant les quatre (4) premiers stades. Selon Bertolucci et al. (2008), la taille, la forme et le volume du foie sont adaptés à l'espace disponible entre les autres organes viscéraux de la cavité abdominale. Quant aux stades V et VI, le volume de l'hépatopancréas est réduit dans la cavité abdominale et est comprimé surtout parles ovaires qui ont augmentés de volume. 


\section{Aspect histologique de l'hépatopancréas}

L'examen histologique de l'hépatopancréas révèle qu'il est constitué $\mathrm{du}$ foie et du pancréas. Par cette association avec le tissu pancréatique, l'organe est désigné hépatopancréas (Roberts, 2001 ; Genten et al., 2011). Selon, ces auteurs, dans le cas de l'hépatopancréas, le tissu pancréatique envahit progressivement le foie durant le développement larvaire. Cependant, il existe certains groupes tels que Chondrichtyens, Serranidae, chez lesquels ces deux glandes digestives restent totalement séparées (Genten et al., 2011). Des résultats similaires ont été obtenus chez le spécimen, Trachurus trachurus Choulika (2002). En effet, au sein d'une même famille, les tissus pancréatiques se combinent tantôt au foie pour former un seul organe compact appelé hépatopancréas, tantôt il reste totalement séparé formant deux organes distincts, le foie et le pancréas. Cependant, d'autres travaux ont montré que les îlots pancréatiques se localiseraient parfois le long de l'intestin chez les poissons zèbres (Pack et al., 1996). Le parenchyme hépatique des Poissons est essentiellement constitué d'hépatocytes non structurés en lobules comme c'est le cas chez les Mammifères où le lobule constitue l'unité fonctionnelle de base (Leeson, 1971; Hould,1982). Chez ces Mammifères, ces deux glandes digestives restent totalement séparées aussi bien au plan anatomique que physiologique.

Les présents résultats se rapportant au foie, montrent l'existence de lames hépatiques disposées autour des capillaires sinusoïdaux, d'artères hépatiques, des veines portes et des centres mélanomacrophages. Ceux-ci confirment les travaux de Biagianti-Risbourg (1990) qui ont montré que la structure du foie des Poissons marins du genre Liza n'apparait pas divisée en lobule et le parenchyme hépatique contient des centres mélanomacrophages. Ces résultats sont similaires également à ceux de Genten et al. (2011).

La présente étude révèle une augmentation du volume des lames hépatocytaires durant les quatre premiers stades. Chaque lame hépatocytaire serait constituée de milliers de cellules hépatocytairesanastomoséesne présentant pas d'organisation spécifique comme décrit par Genten et al. (2011). En effet, selon Genten et al. (2011), chez l'espèce Euchilichthys guentheriles lames hépatocytaires sont moins organisées. Cet aspect histologique des lames hépatocytaires obtenus dans le présent travail est conforme à ceux d'Aarab (2004). Les lames hépatocytaires sont disposées autour des capillaires sinusoïdaux. Des résultats similaires ont été décrits par Meeks et al. (1991). Selon ces derniers, les hépatocytes sont associés les uns aux autres par des jonctions lacunaires et séparés par les capillaires sinusoïdaux.Les vaisseaux sanguins notamment les artères sont dispersées dans le parenchyme hépatique. Ces observations ont été aussi faites par Aarab (2004), chez des espèces de la famille des Cyprinidae (Rutilus rutilus et Perca fluviatilis). Dans le parenchyme hépatique a été observé les 
mélanomacrophages. Cette description corrobore celle de Genten et al. (2011).Varichak (1938), a mentionné que le foie des Poissons ne possède pas de cellules de Kuppfer. Ces résultats sont en accord avec ceux des présents travaux.Aucune structure cellulaire autre que les hépatocytes n'a été mise en évidence.

Quant aux pancréas de Chloroscombrus chrysurus, sa structure ne diffère pas de celle des Mammifères. L'étude de l'hépatopancréas de l'espèce étudiée, a révélé la présence du pancréas exocrine et du pancréas endocrine. Le pancréas exocrine est constitué par des acini séreux et les canaux excréteurs. Le pancréas endocrine est représenté par des amas cellulaires, îlot de Langerhans comme chez les vertébrés supérieurs (Choulika, 2002). En se basant sur les travaux de Beccaria et al. (1990), les îlots de Langerhans contiennent quatre classes de cellules: les cellules A, les cellules B, les cellules D et les cellules PP. Ces quatre types cellulaires n'ont pu être identifiés dans les présents travaux. Car leur mise en évidence demande des colorations topographiques spécifiques. Elle nécessite des techniques cytologiques et des colorantsparticuliers.

La physiologie du pancréas endocrine a été largement étudiée chez les Mammifères. Ainsi,les cellules A assurent la production du glucagon, les cellules B synthétisent l'insuline, les cellules D assurent la sécrétion de la somatostatine et les cellules PP synthétisent le polypeptide pancréatique. (Genten et al., 2011).Selon ces auteurs, l'insuline est une hormone qui provoque une hypoglycémie. Le glucagon augmente la concentration sanguine en glucose par la glycogénolyse au niveau hépatique. Comme c'est le cas chez de nombreuses espèces (Mustomen et al., 2002), le foie du Merlu constitue un organe de stockage des lipides.En revanche, il semble que chez beaucoup de Poissons, l'insuline soit plus impliquée dans le métabolisme protéique (Genten et al., 2011). En effet, certaines cellules de l'îlot de Langerhans existeraient chez les Poissons. L'hypothèse la plus vraisemblable pour expliquer cet état de fait pourrait être l'étude du régime alimentaire,la disponibilité des aliments dans leurs milieux.

\section{Physiologique de l'hépatopancréas}

L'hépatopancréas regorge des cellules qui assurent un rôle de défense (Genten et al., 2011). Chez les Poissons, ce sont les cellules mélanomacrophages et les tissus hématopoïétiques qui assurent la tâche immunitaire. Ces cellules ont été observées dans les présents travaux. Selon ces auteurs, les mélanomacrophages phagocytent diverses substances étrangères et des déchets formant ainsi des phagosomes qui fusionnent avec des vacuoles d'enzymes lytiques ou lysosomes. La grande vacuole ainsi formée constitue le phagolysosome. L'analyse structurale de cet organe durant sa différenciation pourrait s'expliquer par sa physiologie. Sur ce plan, en plus 
d'être un organe de stockage de l'énergie, les hépatopancréas interviendraient dans le métabolisme, la reproductionetla défense.

L'étude de l'hépatopancréas a révélé que le parenchyme hépatique est constitué d'hépatocytes capables d'accumuler des nutriments dans le milieu intra-hépatocytaire. En effet, Mustomen et al. (2002) ontrévélé que le foie représente le lieu de stockage des lipides chez le Merlu. Selon Bauchet (2006), les cellules hépatiques jouent un rôle capital dans le métabolisme des lipides et des protéines. Au niveau du métabolisme des lipides, Thomson et Shaffer (2005), ont mentionné que les cellules hépatiques captent les acides gras, les estérifient, les stockent et aussi synthétisent des phospholipides. Selon Debbal (2005), les hépatocytes synthétisent et secrètent la bile dans les canalicules. Cette dernière est acheminée, accumulée puis stockée dans la vésicule biliaire pour les fonctions digestives.En fonction des besoins de la digestion, elle est libérée dans le canal hépatique et est déversée dans le duodénum. Cette substance non enzymatique facilite leur hydrolyse par les lipases pancréatiques. La présence d'hépatocytes et de canalicules chez les Poissons permettentde supposer qu'ils jouent les mêmes rôles.Une autre fonction du foie est la sécrétion de la bile élaborée par les hépatocytes, stockée puis concentrée dans la vésicule biliaire (Roberts, 2001).

Chez les Poissons, les auteurs tels queHoar (1957) et Bertin et Arambourg (1958) ont démontré que la gamétogenèse nécessite un apport important d'énergie provenantdes proiesconsommées qu'ils stockent essentiellement dans le foie sous forme de lipides.Les cellules hépatiques jouent un rôle essentiel dans la gamétogenèse.Ainsi, les auteurs (Encina \& Granado-Lorencio, 1997 ; Tahari, 2011) ont indiqué que le développement et la maturité ovarienne s'accompagneraient d'une forte demande et dépense énergétique qui seraient stockées essentiellement dans le foie sous forme de lipides. Mustomen et al. (2002) indiquent que la taille des hépatocytes est fonction de sa physiologie. Selon ces auteurs, une hypertrophie cellulaire des lames hépatocytaires serait associée à la vitellogenèse et une hypotrophie cellulaire au jeûne, donc à la reproduction. De plus, Thiaw (1993) a mentionné que les hépatocytes élaborent les réserves vitellines pendant la vitellogenèse qui pénètrent dans les ovocytes par micropinocytose.

Les hépatocytes interviennent aussi dans la défense du poisson grâce à la présence des mélanomacrophages. Ces dernières phagocytent les antigènes, devenant des phagosomes qui fusionnent avec des vacuoles lytiques formant ainsi des phagolysosomes. Ces phagolysosomes s'agrègent entre eux pour former des centres mélanomacrophages (Genten et al., 2011) qui sont de véritables organes d'épuration de l'organisme. Les présentsrésultats se rapprochent de ceux de LeCluyse et al. (2012) qui ont démontré que les hépatocytes jouent un rôle dans l'immunité hépatique puisqu'ils ont une fonction de cellules présentatrices d'antigènes. 


\section{Conclusion}

L'échelle de maturité sexuelle chez Chloroscombrus chrysurus se déroule en six stades durant la croissance du poisson au cours de laquelle s'effectue la différenciation de l'hépatopancréas.

Au plan anatomique, l'hépatopancréas de Chloroscombrus chrysurus est une masse unique. Durant la différenciation de l'hépatopancréas, les critères tels que la mensuration et la colorabilité varient avec les échelles de maturité.

$\mathrm{Au}$ plan histologique, l'hépatopancréas de la femelle de Chloroscombrus chrysurus est un organe mixte constitué du foie et du pancréas. Sa structure histologique révèle qu'elle varie très peu du stade I au stade VI. Au plan structural, le foie est constitué du parenchyme hépatique dans lequel les hépatocytes, les centres mélanomacrophages et des vaisseaux sanguins sont observables. Quant aux pancréas, il est constitué des îlots de Langerhans et des cellules acineuses pancréatiques. Cette architecture est différente de celle des Mammifères, chez lesquels le foie et le pancréas sont deux organes compacts, distincts et séparés. Les Poissons sont des organismes dont l'hépatopancréas semble être à un stade inférieur de différenciation par rapport aux Mammifères. En revanche, la physiologie de ces deux organes n'est pas séparée comme c'est le cas chez les Mammifères. Chez les Poissons, les tissus pancréatiques et hépatiques interviennent dans la reproduction, la digestion et la défense des Poissons.

\section{Remerciements}

Nous tenons à remercier la famille N'GATTA et monsieur KOUA BEIRA GEORGES. Nous exprimons nos sincères remerciements à monsieur KOFFI TANOH, Technicien du GERME à la retraite, et à monsieur SOUMAHORO IBRAHIMA, Doctorant au Laboratoire de Biochimie pour leur aimable collaboration.

\section{References:}

1. Aarab, N. (2004). Les bio marqueurs chez les poissons et Bivalves : de l'exposition à l'effet et du laboratoire au terrain. Thèse de doctorat à l'université de Bordeau I. 278p.

2. Bauchet, E.M. (2006). Réalisation d'un atlas interactif d'histologie topographique du poisson Medaka (Oryziaslatipes). Doctorat vétérinaire à la faculté de médecine de creteil. 98p.

3. Beccaria, C ., Diaz, J.P., Gabrion, J., \& Connes, R. (1990). Maturation du pancréas endocrinien à la loup de mer, Dicentrarchuslabrax L. (Teleostei): étude immunocitochimique et ultrastructurale. I. Cellules produisant du glucagon. Gen. Comp. Endocrinol., 78 (1): 80-92. 
4. Bertolucci, B., Vicentini, C.A., Franceschini-Vicentinii, B., \& Bombonato, M.T.S. (2008). Microscopie optique et ultrastructure du foie d'Astyanax altiparanae (Teleostei, Characidae). Acta Sci. Biol. Sci., 30 (1): 73-6,

5. Biagianti-Risbourg, S. (1990). Contribution à l'étude du foie de juvéniles de nuages (Téléostéens, Mugilidés) contaminés expérimentalement par l'atrazine (s-triazine herbicide): approche ultrastructurale et métabolique ; intérêt en ecotoxicologie. Thèse de Doctorat Es-Sciences Naturelles, Université de Perpignan, 451p.

6. Bodji, I.M., Konan, K.J., Alla, Y.L., \& Atseb, C. (2011). Détermination du sexe chez la carpe blanche Pomadasysjubelili(Valencienne, 1830). F. Tech. \& Doc. Vulg. : 1721.

7. Choulika, M. (2002). Contribution à l'étude de la biologie d'un poisson côtier le SaurelTrachurusTrachurus, (LINNE, 17500) : Anatomie et histologie du tube digestif. Mémoire, 80p.

8. Encina, L. \& Granado-Lorencio, C. (1997). Seasonal changes in condition nutrition, gonad maturation and energy content in Barbel, Barbusscaleteri. Inhabiting a fluctuating river. Environ. Biol. Fish. Vol (50): 75-84.

9. Gabe, M. (1968). Techniques histologiques. Masson et Cie, Éditeurs, 28-262.

10. Genten, F., Terwinghe, E., \& Danguy, A. (2011). Histologie illustrée du poisson. ÉditionsQuae.

11. Hould, R. (1982). Histologie descriptive et éléments d'histopathologie, Décarie éditeurs, Montréal. 148- 151.

12. Humason, G.L. (1967). Animal tissue techniques. W. H. Freeman and

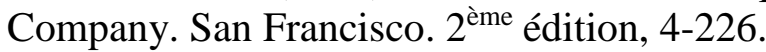

13. Lecluyse, EL., Witek, RP., Andersen, ME., \& Powers, M J. (2012). Organotypicliver culture models: meeting current challenges in toxicitytesting. Critical reviews in toxicology42: 501-48.

14. Leeson, T S. \& Leeson, C R. (1971). Histologie, Masson et C éditeurs. Paris, $451 \mathrm{p}$.

15. Locquin, M. \& Langeron, M. (1978). Manuel de microscopie. Ed. Masson, 2-224.

16. Martoja, R. \& Martoja-Pierson, M. (1967). Initiation aux techniques de l'histologie Animale. Ed.Masson et Cie, 8-125.

17. Meeks, R.G., Harrison, S.D., \& Bull, R. J. (1991). Hepatotoxicology, $700 \mathrm{p}$.

18. Mustonen, AM., Nieminien, P., \& Hyvarinen, H. (2002). Live rand plasma lipids of spawning burbot. J. Fish. Biol(61): 1318-1322. 
19. Nezelof, C., Galle, P., \& Hinglais, N. (1972). Les examens de laboratoires. Techniques microscopiques. Flammarion MédecineSciences, 55-183.

20. Pack, M., Solnica-Krezel, L., Malicki, J., Neuhauss, S. C., Schier, A. F., Stemple, D. L., Driever, W., \& Fishman, M. C. (1996). Mutations affecting development of zebrafish digestive organs, Development. 123, 321-8.

21. Paugy, D. \& Leveque, C. (1999). La reproduction. In : les poissons des eaux continentales africaines: Diversité, Écologie, Utilisation par l'Homme, Lévêque C. et Paugy D. (eds).129- 151.

22. Roberts, R.J. (2001). Fish pathology. 3rd edition Philadelphia: SAUNDERS, 472p.

23. Sylla, S. (2010). Paramètres des populations, biologie de la reproduction et $\mathrm{du}$ régime alimentaire $\mathrm{du}$ CarangidaeTrachinotusteraiaCurvier, 1832 dans la lagune Ebrié (Côte d'Ivoire). Thèse de doctorat de l'Université de Cocody, 128p

24. Tahazi, F. Z. (2011). Contribution à l'étude de la biologie de reproduction d'un petit pélagique le SaurelTrachurustrachurus: Spermatogénèse, Condition, RGS, RHS. Mémoire de MAGISTER, Université d'Oran. 95p

25. Thiaw, O T. (1993). Étude ultrastructurale de la gamétogenèse comparée des Cyprinodontidae Myers, 1955 (poissons, Téléostéens). Relation entre la morphologie des gamètes, la phylogénie et la taxonomie. Doct. D’Etat es Sei. Nat. UniV1. C. A. D. Dakar, 138 p.

26. Thomson, A.B.R. \& Shaffer, E.A. (2005). Principes fondamentaux de gastro-entérologie États pathologiques et démarches thérapeutiques. $972 \mathrm{p}$.

27. Varichak, T. (1938). Studies on endothelialcells in the liver of fishes. Z. Zellforsh. Mikrosk. Anat., 4, 46-51. 